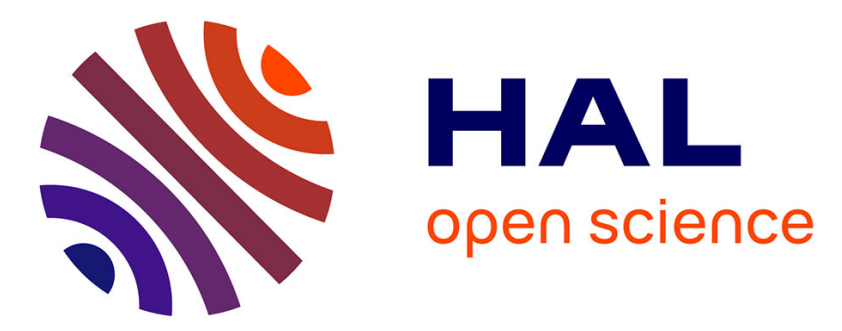

\title{
On the hybrid aid-localization for outdoor augmented reality applications
}

Imane Zendjebil, Fakhr-Eddine Ababsa, Jean-Yves Didier, Malik Mallem

\section{To cite this version:}

Imane Zendjebil, Fakhr-Eddine Ababsa, Jean-Yves Didier, Malik Mallem. On the hybrid aidlocalization for outdoor augmented reality applications. VRST '08: Proceedings of the 2008 ACM symposium on Virtual reality software and technology, Oct 2008, Bordeaux, France. pp.249-250, 10.1145/1450579.1450637. hal-00344877

\section{HAL Id: hal-00344877 \\ https://hal.science/hal-00344877}

Submitted on 9 Nov 2011

HAL is a multi-disciplinary open access archive for the deposit and dissemination of scientific research documents, whether they are published or not. The documents may come from teaching and research institutions in France or abroad, or from public or private research centers.
L'archive ouverte pluridisciplinaire HAL, est destinée au dépôt et à la diffusion de documents scientifiques de niveau recherche, publiés ou non, émanant des établissements d'enseignement et de recherche français ou étrangers, des laboratoires publics ou privés. 


\title{
On the Hybrid Aid-Localization for Outdoor Augmented Reality Applications
}

\author{
I.M. Zendjebil $^{*} \quad$ F. Ababsa ${ }^{\dagger} \quad$ J-Y. Didier ${ }^{\ddagger} \quad$ M. Mallem ${ }^{\S}$ \\ IBISC Laboratory, University of Evry Val d'Essonne, CNRS FRE 3190
}

\begin{abstract}
In mobile outdoor augmented reality applications, accurate localization is critical to register virtual augmentations over a real scene. Vision-based approaches provide accurate localization estimates but are still too sensitive to outdoor conditions (brightness changes, occlusions, etc.). This drawback can be overcome by adding other types of sensors. In this work, we combine a GPS and an inertial sensor with a camera to provide accurate localization. We will present the calibration process and we will discuss how to quantify the 3D localization accuracy. Experimental results on real data are presented.
\end{abstract}

CR Categories: K.6.1 [Infomation Systems]: Outdoor Augmented Reality—; K.7.m [Computer Vision]: 3D localizationHybrid sensor Calibration

Keywords: Outdoor augmented reality, 3D localization, hybrid sensor, calibration, error prediction

\section{Introduction}

Vision-based methods are widely used in augmented reality (AR) applications to obtain an accurate registration. In outdoor scenes, these types of methods lack of robustness and accuracy mainly due to outdoor conditions. Adding other types of sensors improves vision-based approaches. Indeed, we can combine GPS to initialize visual tracking [Reitmayr and Drummond 2007], fuse inertial sensor data with a visual tracking [Bleser and Stricker 2008] to improve stability and robustness, or assist visual tracking with inertial sensor [Aron et al. 2007].

In our work, we combine a GPS with an inertial sensor to assist a visual tracking process. The combination of GPS and inertial sensor represent our Aid-localization system. This combination is used to estimate continuously the user's position and orientation even when vision tracking fails. The accuracy of this hybrid sensor depends on the accuracy of the calibration procedure that determines the relationship between the different used references frames. We propose two calibration processes to estimate the relationship between inertial sensor and camera and the transformation which maps the GPS position to the camera position. The measures provided by the Aid-

\footnotetext{
*e-mail:maissa.zendjebil@ibisc.fr

${ }^{\dagger}$ fakhreddine.ababsa@ibisc.fr

¥jean-yves.didier@ibisc.fr

§malik.mallem@ibisc.fr
}

localization module are less accurate. So, we need to quantify these errors in order to correct the estimated position and orientation.

\section{Calibration Procedure}

Each sensor provides data in its own reference frame. The inertial sensor computes the orientation between a local inertial reference frame $\mathscr{R}_{I}$ attached to itself and a global inertial reference frame $\mathscr{R}_{G}$. The GPS's position is expressed in a global reference frame. For registration, we need to estimate continually the camera's pose which relates the world reference frame $\mathscr{R}_{W}$ and the camera reference frame $\mathscr{R}_{C}$. Thus, the pose provided by the Aid-localization system must be aligned with the camera reference frame $\mathscr{R}_{C}$.

\subsection{Inertial/Camera Calibration}

We need to estimate the rotation between reference frames attached to the camera and the inertial sensor in order to transpose the orientation obtained from one sensor into the reference frame of the other. We define $R_{C W}$, obtained from the camera pose estimation, the rotation of the world reference frame $\mathscr{R}_{\mathscr{W}}$ with respect to the camera reference frame $\mathscr{R}_{C}$. The rotation of the local inertial reference frame $\mathscr{R}_{I}$ with respect to $\mathscr{R}_{C}$ is $R_{C I}$. Also, $R_{I G}$ is the rotation between $\mathscr{R}_{I}$ and the global inertial reference frame $\mathscr{R}_{G}$ provided by inertial sensor. The rotation between the $\mathscr{R}_{W}$ and $\mathscr{R}_{G}$ is defined by $R_{G W}$. Thus, the transformation between the camera and inertial sensor is defined by $R_{C I}$ and $R_{G W}$ and expressed as:

$$
R_{C W}=R_{C I} R_{I G} R_{G W}
$$

In our case, the Inertial/Camera calibration process consists in estimating a rotation matrix $R_{C I}$ and deducing the second rotation matrix $R_{G W}$. We assume that the $Z$ axis of the global inertial reference frame $\mathscr{R}_{G}$ is pointing up along vertical. To simplify the equation (1), we define the $Z$ axis of the world reference frame collinear with it ( $Z$ axis is along vertical and pointing up). Therefore, $R_{G W}$ is only a rotation around the $Z$ axis with an angle $\theta$. With this configuration, we estimate $R_{C I}$ using least mean squares from the following equation:

$$
r_{3}^{C W}=R_{C I} r_{3}^{I G}
$$

With $r_{3}^{C W}$ and $r_{3}^{I G}$ are the third column of $R_{C W}$ and $R_{I G}$ respectively. Then, $R_{G W}$ is deduced using (1):

$$
R_{G W}=R_{I G}^{T} R_{C I}^{T} R_{C W}
$$

\subsection{GPS/Camera Calibration}

The GPS/Vision calibration estimates the rigid transformation (rotation + translation) which allows to deduce the camera's position from the position provided by the GPS. For each GPS position $p_{g p s}$, we associate a camera's position $p_{\text {cam }}$ obtained from the estimated pose $\left(p_{c a m}=-R_{C W}^{T} t_{C W}\right)$. The relationship between the two positions is given by: $p_{\text {cam }}=R p_{\text {gps }}+t$. The rigid transformation is obtained by minimizing the following criterion:

$$
\sum_{i}^{n}\left\|p_{c a m}^{i}-R p_{g p s}^{i}+t\right\|^{2}
$$


We introduce then the vectors $\vec{N}_{g p s}^{i}=p_{g p s}^{j}-p_{g p s}^{i}$ and $\vec{N}_{c a m}^{i}=$ $p_{\text {cam }}^{j}-p_{\text {cam }}^{i}$ with $i=1 . . \frac{n}{2}$ and $j=\frac{n}{2}+1 . . n$.. The relationship deduced from (4) between them is:

$$
\sum_{i}^{\frac{n}{2}}\left\|\vec{N}_{c a m}^{i}-R \vec{N}_{g p s}^{i}\right\|^{2}
$$

From equation (5), we can estimate the rotation $R$. The translation is then deduced from the following equation (With $\bar{p}_{\text {cam }}=\frac{1}{n} \sum_{i}^{n} p_{c a m}^{i}$ and $\left.\bar{p}_{g p s}=\frac{1}{n} \sum_{i}^{n} p_{g p s}^{i}\right)$ :

$$
t=\bar{p}_{c a m}-R \bar{p}_{g p s}
$$

We use GPS to obtain a 2D localization (longitude and latitude). Concerning the altitude, we fixed it according to where we are in the environment.

\section{Error Prediction}

The error estimation is important in the localization process. Indeed, it allows quantifying the quality of measurements and improving the estimate provided by the aid-localization module. Our error represents the difference with respect to the camera's pose provided by vision method. When the vision fails, we need to predict this error. So, we opt for a regression with a Gaussian process [Williams 1997]. A Gaussian process is a stochastic process which generates samples and can be used as a prior probability distribution over functions in Bayesian inference. During visual tracking, we record the offset between the hybrid sensor and camera pose. When the visual tracking fails, the Gaussian process allows predicting the offset made by hybrid sensor and improves the localization estimate.

\section{Experiments and Results}

We use data acquired in an outdoor environment to calibrate the different sensors and validate the 3D localization using GPS and inertial sensor. The camera's pose used to calibrate the hybrid sensor is estimated using a manual $2 \mathrm{D} / 3 \mathrm{D}$ points matching (for accurate estimate). The camera poses are estimated along the images sequence using a point-based visual tracking and was used as ground truth. To validate Inertial/Camera Calibration, different views were taken with various rotation angles. For each view, we estimated the camera's rotation using inertial orientation and vision method. The means errors obtained between the two estimated rotations are shown in table 1. The use of inertial sensor allows us to cover the camera's orientation with low errors. This represents a good result for registration. Concerning position, the transformation between

\begin{tabular}{|c|c|c|c|}
\hline & $\phi$ & $\theta$ & $\psi$ \\
\hline Mean error & $-0.3460^{\circ}$ & $-0.7211^{\circ}$ & $-2.293^{\circ}$ \\
\hline
\end{tabular}

Table 1: Angular means errors in degree

the GPS and the camera was estimated using a set of fixed images (20 images) with geo-referenced coordinates provided by the GPS. The different positions are well spread over the work area. Thereafter, we tested the validity of the calibration procedure on a set of data taken continuously. Figure 1 and table 2 show the errors obtained on meters (red line). The obtained results are satisfactory for the purposes of the application (i.e. to assist visual tracking). The GPS position can move closer to the real position using the predicted errors. In our experimentation, predicted error is estimated with a set of $N=40$ last sample (empirically determined). We can see the predicted errors in figure 1 (green line) and table 2.

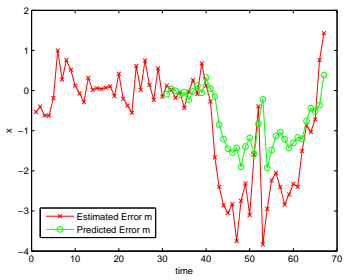

(a) On $x$ axis

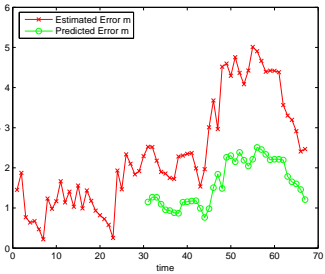

(b) On y axis
Figure 1: Estimated Error vs. Predicted Error

\begin{tabular}{|c|c|c|c|c|}
\hline Errors & $\mu_{x}$ & $\sigma_{x}^{2}$ & $\mu_{y}$ & $\sigma_{y}^{2}$ \\
\hline Estimated & $0.7572 m$ & $1.7087 m^{2}$ & $2.344 m$ & $1.8473 m^{2}$ \\
\hline Predicted & $0.8604 m$ & $0.4588 m^{2}$ & $0.9015 m$ & $0.3174 m^{2}$ \\
\hline
\end{tabular}

Table 2: Mean errors and variation in meters

\section{Conclusion}

In this work, we present our hybrid sensor composed of a camera coupled with a GPS and an inertial sensor. The use of the GPS and the inertial sensor provide an estimate of the camera's pose when visual tracking fails. For this, we propose two calibration processes allowing to deduce the camera's pose from data provided by these two other sensors. Our calibration processes are distinguished by its simplicity and efficiency. In addition, our methods do not require heavy assumptions. Also, to characterize the quality of the localization of the system, the use of Gaussian process allows predicting the obtained error and thence improving the estimate. The results obtained are quite satisfactory.

\section{Acknowledgements}

This work is supported by the RAXENV project funded by the French National Research Agency "ANR".

\section{References}

Aron, M., Simon, G., ANd Berger, M. 2007. Use of inertial sensors to support video tracking: Research articles. Comput. Animat. Virtual Worlds 18, 1, 57-68.

Bleser, G., And Stricker, D. 2008. Advanced tracking through efficient image processing and visual-inertial sensor fusion. In $V R, 137-144$.

Reitmayr, G., AND DRUmmond, T. 2007. Initialisation for visual tracking in urban environments. In IEEE ISMAR.

Williams, C. 1997. Prediction with gaussian processes: From linear regression to linear prediction and beyond. Tech. rep., Neural Computing Research Group, Oct. 